\title{
PREVALENCIA DE SOBREPESO/OBESIDAD Y FACTORES DE RIESGO ASOCIADOS EN EMPLEADOS DEL FONDO DE PROTECCIÓN DE LISIADOS Y DISCAPACITADOS A CONSECUENCIA DEL CONFLICTO ARMADO, ENERO-ABRIL DE 2017
}

Delmy Virginia Granados Castro ${ }^{1}$ Elmer Wilfredo Mendoza Rodríguez ${ }^{2}$ Isis Stephanie Rivas Hernández ${ }^{3}$ Recibido: 25/05/17 Aceptado: 04/12/17

DOI: http://dx.doi.org/10.5377/creaciencia.v11i1-2.6038

\section{RESUMEN}

OBjetivo. El objetivo de este estudio fue determinar la prevalencia de sobrepeso/obesidad y factores de riesgo asociados en empleados del Fondo de Protección de Lisiados y Discapacitados a Consecuencia del Conflicto Armado (FOPROLYD), así como determinar la prevalencia de obesidad central. Metodología. Estudio descriptivo transversal de corte analítico en el que se estudió a 230 personas (con un 93\% respuesta), independientemente de si son discapacitados físico o no. Se tomaron medidas antropométricas: peso, talla y circunferencia abdominal, ajustando el peso en el caso de personas con algún miembro amputado, se obtuvo el índice de masa corporal y se entrevistó a cada uno de ellos; finalmente, la asociación estadística de factores de riesgo con la enfermedad. Resultados. Se encontró una prevalencia global del $83.50 \%$ de sobrepeso/obesidad a predominio de obesidad; además, un elevado porcentaje de hábitos de alimentación inadecuados, sedentarismo y antecedente familiar de obesidad. Se encontró que una de cada tres personas posee obesidad central. Los factores de riesgo que se asociaron con obesidad fueron el consumo de más de cinco bebidas azucaradas procesadas a la semana, antecedente familiar de obesidad, peso anormal al nacer y haber padecido obesidad en etapas tempranas. ConclusioNES. Hay una elevada prevalencia de sobrepeso y obesidad en hombres y mujeres. Los factores de riesgo no modificables en la etapa adulta tienen significativamente mayor influencia en el padecimiento de obesidad que los factores modificables.

Palabras clave: Sobrepeso/obesidad, lisiados, discapacitados, foprolyd, El Salvador. 


\title{
PREVALENCE OF OVERWEIGHT/OBESITY AND ASSOCIATED RISK FACTORS IN EMPLOYEES OF THE FONDO DE PROTECCIÓN DE LISIADOS Y DISCAPACITADOS A CONSECUENCIA DEL CONFLICTO ARMADO, JANUARY-APRIL 2017
}

\begin{abstract}
ОвJective. The objective of this study was to determine the prevalence of overweight/obesity and associated risk factors in employees of the Fondo de Protección de Lisiados y Discapacitados a Consecuencia del Conflicto Armado (FOPROLYD), and to determine the prevalence of central obesity. Methodology. A cross-sectional descriptive study of 230 patients (with $93 \%$ response) with or without physical disability. Anthropometric measures were taken: weight, height and waist circumference, adjusting the weight in people with an amputated limb, obtaining the body mass index and interviewing each one of them; finally, the statistical association of risk factors with the disease. Results. Overall $83.50 \%$, mostly, obesity; in addition a high percentage of inadequate eating habits, sedentary lifestyle and family history of obesity. It was found that 1 in 3 people have central obesity. The risk factors associated with obesity were to consume more than 5 sugary drinks processed per week, a family history of obesity, abnormal birth weight, and obesity in the early stages. Conclusions. There is a high prevalence of overweight and obesity in men and women. Non-modifiable risk factors in adulthood have a significantly greater influence on obesity than those modifiable factors.
\end{abstract}

KeYwords: Overweight /obesity, disabled, FOPROLYD, El Salvador.

\section{INTRODUCCIÓN}

El sobrepeso y la obesidad han alcanzado proporciones epidémicas a nivel mundial, por lo que constituyen un grave problema de salud pública. ${ }^{1}$ Se trata de un fenómeno que afecta tanto a países de altos ingresos como a los de renta media y baja, a predominio del área urbana., ${ }^{2,3}$ En América Latina, Chile y México presentan los índices más elevados ${ }^{3-4}$. En El Salvador, según la «Encuesta Nacional de Salud Familiar FESAL 2008» y la «Encuesta Nacional de Enfermedades Crónicas no Transmisibles en Población Adulta de El Salvador ENECA-ELS 2015», se evidencia un aumento reportado del $60 \%$ al $65 \%$ para sobrepeso y obesidad en conjunto. ${ }^{5,6}$ FOPROLYD no dispone de datos específicos, por lo que con este estudio se marca una línea de base.

\section{MÉTODO}

\section{Población}

Estudio de base poblacional en el que, de 250 personas elegibles (117 mujeres y 133 hombres), se estudiaron 230 (106 mujeres y 124 hombres), quienes documentaron su voluntad de participar mediante la firma de consentimiento informado. No se logró estudiar a catorce personas por dificultades de accesibilidad, se excluyó a dos mujeres por encontrarse en estado de gestación y cuatro se rehusaron a participar, lo que dejó un 93\% de validez.

\section{Mediciones y recolección de datos}

Para la toma de medidas antropométricas a cada participante se procedió de esta manera: para el 
peso $(\mathrm{kg})$, con la menor cantidad de objetos pesados, sin zapatos y con ropa ligera; para la talla (m), con la espalda recta y la vista al frente; para la circunferencia abdominal $(\mathrm{cm})$, con cinta métrica flexible no extensible en el punto medio entre la espina ilíaca anterosuperior y la última costilla. Se calculó el índice de masa corporal (IMC) mediante el cociente del peso $(\mathrm{kg})$ entre la talla elevada al cuadrado $\left(\mathrm{m}^{2}\right)$. Posteriormente, se entrevistó a cada uno de ellos aplicando un cuestionario para conocer los factores de riesgo biológicos y conductuales.

\section{Análisis}

Se clasificó el estado nutricional de acuerdo con los criterios establecidos en el consenso SEEDO ${ }^{7}$. Se definió como caso (sobrepeso/obesidad) a aquella persona cuyo IMC fue mayor a $25\left(\mathrm{~kg} / \mathrm{m}^{2}\right)$. Se realizó ajuste del peso a personas con algún miembro amputado mediante los criterios de Osterkamp ${ }^{8}$, tomando en cuenta el nivel de amputación.

Debido a que la acumulación de grasa abdominal se relaciona con mayor riesgo cardiovascular, se determinó obesidad central según los criterios de la ATP III cuando es igual o mayor de $88 \mathrm{~cm}$ para el sexo femenino y $102 \mathrm{~cm}$ para el masculino. . $^{7,10}$

Existen múltiples factores de riesgo para padecer sobrepeso/obesidad incluso desde edades tempranas, factores que al persistir en el tiempo se relacionan con otras enfermedades crónicas. ${ }^{11,12}$ En cuanto a los factores conductuales, se tomó en cuenta la calidad de alimentación. Debido a que no es recomendable limitar ni saltarse tiempos de comida, ${ }^{13-15}$ se preguntó por la cantidad de comidas ingeridas en un solo día, teniendo en cuenta que el límite adecuado es de cinco tiempos diarios. En cuanto al consumo de frutas y verduras, se consideró como factor de riesgo comer menos de cinco porciones entre ambos componentes al día; ${ }^{11}$ además, se tomó en cuenta el consumo diario de frutas y vegetales en la última semana y el tipo de grasa utilizado para cocinar (aceite, manteca, margarina y mantequilla). La actividad física se catalogó como aquellas personas que realizan actividad física, ya sea caminata, intensidad moderada o vigorosa al menos tres días de la última semana de acuerdo a los criterios del IPAQ, ${ }^{16}$ medidos en equivalentes metabólicos del consumo de oxígeno (MET) ${ }^{11}$ El consumo de bebidas azucaradas se determinó de acuerdo con el hecho de haber consumido al menos una bebida estándar en la última semana. ${ }^{11}$ Respecto a los factores perinatales, se consideró factor de riesgo el parto abdominal, ${ }^{17,18} \mathrm{el}$ peso anormal al nacer, ya sea alto o bajo, y el parto prematuro ${ }^{19-22}$. En los antecedentes familiares (madre, padre y hermanos), se consideró como factor de riesgo, el tener antecedente de obesidad de alguno de ellos; ${ }^{23-25}$ y el antecedente personal de haber padecido obesidad en etapas tempranas como la infancia y la adolescencia. ${ }^{26}$

El análisis y descripción se realizó mediante tablas de frecuencia y cálculo de Chi cuadrada con Epi Info 3.5.4 para la asociación de la enfermedad (obesidad) con los factores de riesgo.

\section{Resultados}

La población estudiada se compone de $54 \%$ de hombres y $46 \%$ de mujeres, de los cuales $7 \%$ ha sufrido amputación de algún miembro. La mayoría se encuentra entre 30 y 39 años de edad, y entre 40 y 49.

La prevalencia total (Tabla 1) de sobrepeso/obesidad fue del $83.50 \%$ (IC: $78 \%-88 \%$ ), $40.9 \%$ de sobrepeso y $42.6 \%$ de obesidad. En la distribución por estados nutricionales (Figura 1) se puede observar que los dos estados de mayor prevalencia son la obesidad grado I y la preobesidad, para ambos sexos.

La distribución por edad fue entre 20 a 39 años, el IMC fue menor a $27 \mathrm{~kg} / \mathrm{m}^{2}$; sin embargo el IMC aumenta a partir de los 40 años para ambos sexos y el pico de obesidad grado II se encuentra entre 50 a 59 años de edad.

El 37\% de la población presentó obesidad central, con una distribución similar en ambos sexos: $18.70 \%$ en mujeres y $18.30 \%$ en hombres. 
Tabla 1. Prevalencia de sobrepeso/obesidad $(\mathrm{N}=230)$

\begin{tabular}{lccc}
\hline Prevalencia & $\begin{array}{c}\text { Valor } \\
\text { ponderado }\end{array}$ & \multicolumn{2}{c}{$\begin{array}{c}\text { 95\% intervalo de } \\
\text { confianza }\end{array}$} \\
& & Inferior & Superior \\
\hline $\begin{array}{l}\text { Sobrepeso/ } \\
\text { obesidad }\end{array}$ & $83.50 \%$ & $78.00 \%$ & $88.00 \%$ \\
Peso normal & $16.50 \%$ & $12.00 \%$ & $22.00 \%$ \\
\hline Total & $100.00 \%$ & & \\
\hline
\end{tabular}

Fuente: Base de datos ESOBFE-FOPROLYD

Relativo al consumo de frutas y verduras, una de cada tres personas afirma incluir al menos una porción en su alimentación diaria.

Sin embargo, el 78.70\% (IC: 72.80\%-83.80\%) consume menos de cinco porciones en el mismo día, con una distribución similar en ambos sexos.

En cuanto a la ingesta de alimentos procesados de preparación rápida (chatarra), el 30\% de los hombres afirma consumirlos una vez a la semana y el $35 \%$ de mujeres, dos veces a la semana, en promedio. El 96.8\% de la población refiere que los alimentos que ingiere son cocinados con aceite vegetal.

La población que toma al menos cinco tiempos de comida al día es el $17.4 \%$. En cuanto al consumo de bebidas azucaradas, el 31.3\% no tomó ninguna en la última semana; sin embargo hay un 14\% que ingiere más de siete.

Por otro lado, en cuanto a la actividad física y el sedentarismo, el $74 \%$ permanecen sentados entre seis y diez horas, e incluso el 15\% más de diez horas en un solo día. Hay un $60.9 \%$ de sedentarismo (Figura 2) en la población estudiada, mientras que la distribución por sexo de quienes sí se ejercitan (alcanzando al menos $600 \mathrm{~m} / \mathrm{min} / \mathrm{semana}$ ) es similar en hombres y mujeres (una de cada dos personas practica actividad física).

A la consulta sobre los antecedentes familiares de obesidad, el 48.26\% respondió afirmativamente; de este porcentaje, el $63.93 \%$ refiere que sus padres, o al menos uno de ellos, adolecen de obesidad.

En los antecedentes perinatales, el $10.9 \%$ refiere haber nacido por vía abdominal, un 5.7\% nació antes de que el embarazo llegara a término y el $14.80 \%$ manifiesta que su peso al nacer fue anormal, ya sea alto o bajo.

El 29.10\% de la población estudiada padeció obesidad en la infancia o en la adolescencia.

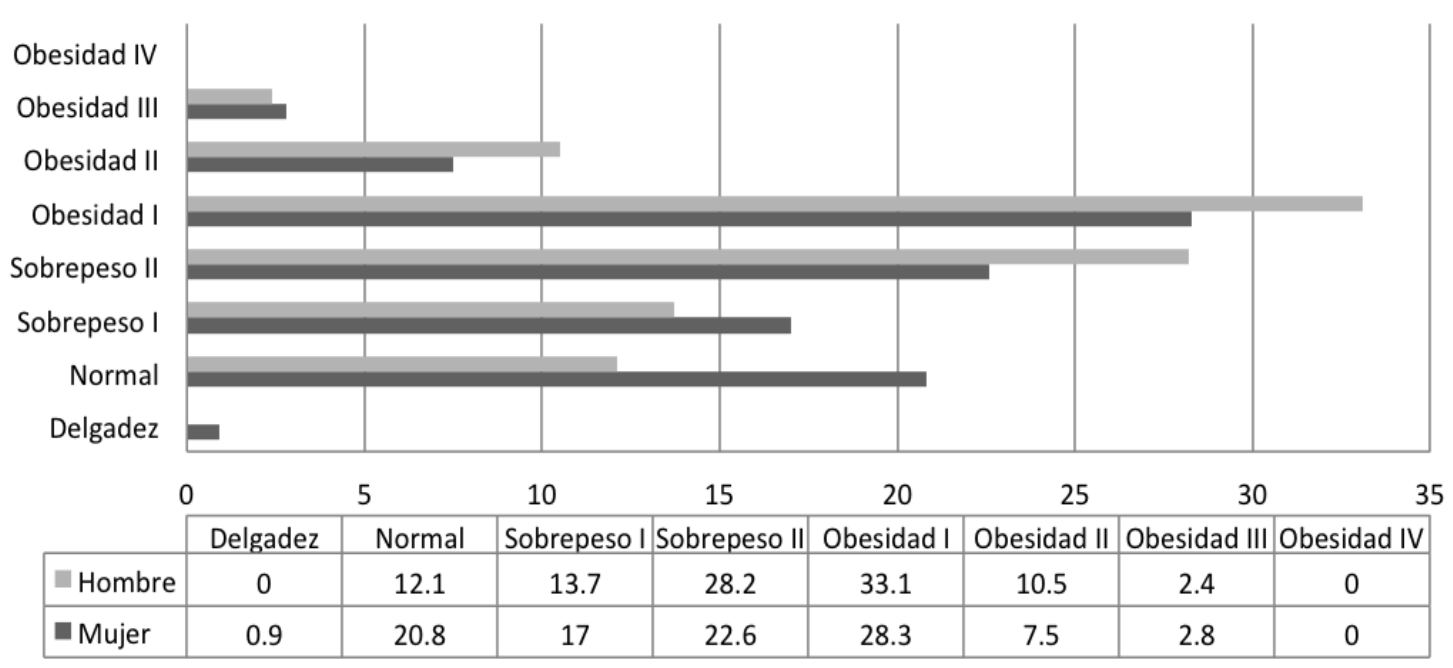

Figura 1. Distribución del estado nutricional por género. 


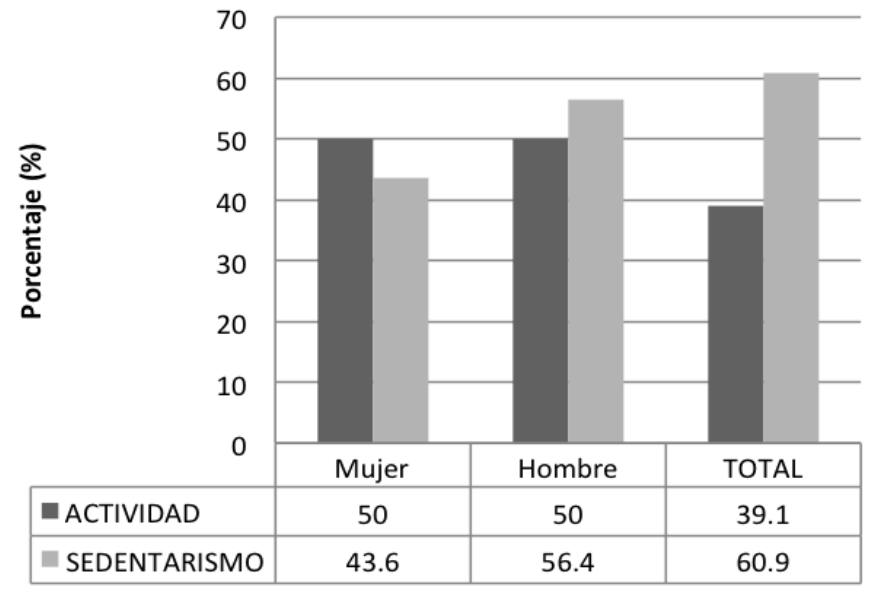

Sin embargo, en el estudio ESOBFEFOPROLYD la obesidad es mayor en hombres, a diferencia de la ENECA y de la ESANUT 2012, donde se encontró más en mujeres. El sobrepeso es similar en ambos sexos en los resultados de estos estudios.

Además, una de cada tres personas estudiadas padece obesidad central, lo cual predispone a comorbilidades tales como enfermedades cardiovasculares; la ESANUT 2012 reportó un $73.9 \%$ de obesidad central. ${ }^{29}$
Figura 2. Prevalencia de actividad física y sedentarismo distribuido por género.

Los factores de riesgo asociados con obesidad fueron: consumir más de cinco bebidas azucaradas procesadas a la semana (p: 0.014, RR: 1.41, IC: 1.02-1.95); antecedente familiar de obesidad (p: 0.0005, RR: 1.75, IC: 1.27-2.41); peso anormal al nacer (p: 0.001; RR: 1.63, IC: 1.17-2.28) y haber padecido obesidad infantil o en la adolescencia (RR: 1.36, IC: 1.00-1.84). No se encontró asociación en este estudio con el resto de factores detallados: el consumo inadecuado de frutas y vegetales, el sedentarismo, la vía del parto ni el parto prematuro.

\section{Discusión}

La prevalencia de sobrepeso/obesidad encontrada en este estudio fue del 83.5\% (40.90\%: sobrepeso y 42.60\%: obesidad), más alta que la que se reporta en la ENECA-ELS 2015, donde se encontró un 37.9\% de sobrepeso y $27.3 \%$ de obesidad (en conjunto, $65.2 \%{ }^{6}$ ); esto es más elevado que lo encontrado en la FESAL $(37 \% \text { y } 23 \% \text {, respectivamente) })^{5}$ y que la reportada por el INCAP en entrevista de prensa $(60 \%$ en conjunto) ${ }^{28}$. Este dato supera a los reportados en un grupo de personas estudiadas en Lima, Perú, donde se encontró $33.7 \%$ de sobrepeso y $14.4 \%$ de obesidad $^{3}$, y lo que México reporta en la Encuesta Nacional de Salud y Nutrición-ESANut 2012: 71\% de sobrepeso y obesidad. ${ }^{29}$
Entre los factores de riesgo conductuales, el bajo consumo de frutas y verduras en la población estudiada fue menor que el encontrado en la ENELCA-ELS 2015 (78.70\% y 93.8\%, respectivamente). Si bien en otros estudios este factor se ha visto relacionado, en este no se encontró asociación estadística.

Al igual que el consumo de bebidas azucaradas, en los datos encontrados en este estudio la prevalencia es menor que el reportado a nivel nacional, aunque ambos datos están elevados (81\% para la ENELCA-ELS 2015 y 68.6\% en FOPROLYD). Sin embargo, el mero hecho de consumir bebidas azucaradas, al parecer, no es problema, sino su cantidad y su frecuencia, dado que sí se encontró significancia estadística con el consumo mayor a cinco bebidas estándar durante la misma semana.

El sedentarismo es un problema a nivel de la institución, ya que el $61 \%$ no realiza actividad suficiente para gastar las calorías consumidas. A nivel nacional, el dato encontrado fue del $40 \%$, lo cual confirma que es un problema no solamente local, sino de país. Si bien el gasto energético mediante el ejercicio físico contribuye a quemar las calorías consumidas, no se encontró asociación estadística entre el hecho de no realizar actividad física con el aparecimiento de la enfermedad.

En este estudio, el 29.10\% tiene el antecedente personal de haber padecido obesidad en su infancia. Si 
bien no se tienen otros datos en población adulta en el país, la Encuesta Mundial de Salud Escolar 2013 reportó que un $38.4 \%$ presenta problemas de sobrepeso y obesidad $(28.8 \%$ y $9.6 \%$ respectivamente), sin diferencia por edad, sexo o grado. ${ }^{30}$ Estos valores cercanos sugieren que las personas que padecieron obesidad en etapas tempranas tienen más probabilidad de continuar con este padecimiento en etapas posteriores.

Como último punto, el $82.6 \%$ realiza menos de cinco tiempos de comida al día, tal como se reporta en un estudio efectuado en la Universidad del País Vasco, en el que $83.6 \%$ realizaban menos de cinco comidas diarias. ${ }^{15}$ No obstante, este factor es limitado, ya que es de tomar en cuenta no solamente la cantidad de tiempos de comida al día, sino la calidad de alimentos y la cantidad de las porciones servidas, es decir, una dieta controlada.

\section{CONCLUSIONES}

La prevalencia de sobrepeso/obesidad encontrada en los empleados de FOPROLYd es elevada en ambos sexos, con tendencia a aumentar de manera proporcional a la edad. La prevalencia de obesidad central es elevada en toda la población, independientemente de su peso. Los factores de riesgo no modificables en la etapa adulta ejercen una influencia significativamente mayor para padecer obesidad que aquellos factores modificables.

\section{REFERENCIAS BIBLIOGRÁFICAS}

1. wHo, World Health Organization. Obesity and overweight. World Health Organization. 2015 January: p. 311.

2. who, World Health Organitazion. 10 facts on Obesity. World Health Organitazion. 2013 May.

3. Lozano-Rojas, G.; Cabello-Morales, E.; HernánDez-Díaz, H.; Loza-Munarriz, C.; «Prevalencia de sobrepeso y obesidad en adolescentes de un distrito urbano, Lima, Perú». Revista Peruana de Medicina Experimental y Salud pública. 2012; 31.
4. Fleming, T.; Robinson, M.; Thomson, B.; Graetz, N.; Margono, C.; et ál. «Global, regional and national prevalence of overweight and obesity in children and adults during 1980-2013: a systematic analysis for the Global Burden of Disease Study 2013». The Lancet. 2014; 384(9945): 755-81. Available in: http://www.thelancet.com/ pdfs/journals/lancet/PIIS0140-6736(14)604608.pdf

5. MSPAS-USAID-UNICEF-UNFPA-OPS. «Datos epidemiológicos sobrepeso y obesidad». Encuesta Nacional de Salud Familiar (FESAL). San Salvador: 2008.

6. Ministerio de Salud/Instituto Nacional de Salud. Encuesta Nacional de Enfermedades Crónicas no Transmisibles en Población Adulta de El Salvador ENECA-ELS 2015. Resultados relevantes. San Salvador, El Salvador: Ministerio de Salud e Instituto Nacional de Salud; 2015.

7. Rubio, M. A.; Salas-Salvadó, J.; Barbany, M.; Moreno, B.; Aranceta, J.; Bellido, D.; et ál. «Consenso SEEDo 2007 para la evaluación del sobrepeso y la obesidad y el establecimiento de criterios de intervención terapéutica». Revista Española de Obesidad [en línea]. Barcelona, España. 2007; 128(5): p. 184-96. Disponible en: https://www.seedo.es/images/site/documentacionConsenso/Consenso_SEEDO_2007.pdf

8. Osterkamp, L. K. «Current Perspective on Assessment of Human Body Proportions of Relevance to Amputees». Journal of the American Dietetic Association. 1995 Feb.; 95(2).

9. Kuh, D.; Hardy, R.; Chaturvedi, N.; WadswORTH, M. E. J. «Birth weight, childhood growth and abdominal obesity in adult life». International Journal of Obesity. 2002 January; 26(1): p. 40-7.

10. Adult Treatment Panel III (ATP III). «Expert Panel on Detection, Evaluation, and Treatment of High Blood Cholesterol in Adults» [on line]. National Cholesterol Education Program (NCEP). 2002 Sep- 
tember; 285:2486-97. Available in: https://www. nature.com/articles/0801861.pdf

11. Organización Mundial de la Salud (oms). Método STEPwise para la vigilancia de los factores de riesgo de las enfermedades crónicas. Ginebra, Suiza: oms; 2006.

12. Dietz, W. H. «Critical periods in childhood in developement of obesity» [on line]. The Americal Journal of Clinical Nutricion. 1994 May; 59(9559). Available in: https://academic.oup.com/ajcn/ article/59/5/955/4732557

13. Patterson, R. E.; Haines, P. S.; Popkin, B. M.; «Diet quality index: capturing a multidimensional behavior»[on line]. Journal of the American Dietetic Association. 1994; 94:57-64. https://www. ncbi.nlm.nih.gov/pubmed/8270756

14. 14. Martínez, J. A.; Navas-Carretero, S.; Saris, W. H.; Astrup, A. «Personalized weight loss strategies-the role of macronutrient distribution. »[on line]Nature Review. Endocrinology. 2014; 1012(749-60). Available in: https://www.ncbi. nlm.nih.gov/pubmed/25311395

15. Arroyo Izaga, M; Rocandio Pablo, A. M.; Ansotegui Alday, L;; Pascual Apalauza, E; Salces Beti, I.; Rebato OchoA, E. «Calidad de la dieta, sobrepeso y obesidad en estudiantes universitarios». Nutrición Hospitalaria [en línea], 2006; 21(6): p. 673-79. Disponible en: http://scielo.isciii.es/pdf/nh/v21n6/original6.pdf

16. IPAQ Research Committee. Guidelines fot he data processing and Analisis of the international Physical Activity Questionnaire. 2005 November.

17. Aguilar Cordero, M. J.; Sánchez López, A. M.; Madrid Baños, N.; Mur Villar, N.; Expósito Ruiz, M.; Hermoso Rodríguez, E. «Lactancia materna como prevención de sobrepeso y obesidad en el niño y adolescente: revisión sistemática». Nutrición Hospitalaria. 2015; 31. Disponible en: http://www.aulamedica.es/nh/pdf/8458.pdf
18. Domínguez-Bello, M. G.; Costello, E. K.; Contreras, M.; Magris, M.; Hidalgo, G.; FierER, N.; KNIGHTB, R. «Delivery mode shapes the acquisition and structure of the initial microbiota across multiple body habitats in newborns». PubMed. 2010; 107(26): p. 11971-75.

19. Loaiza, S.; Coustasse, A.; Urrutia-Rojas, X.; Atalah, E. «Birth weight and obesity risk at first grade in a cohort of Chilean children». Nutrición Hospitalaria. 2011 Febrero; 26(1).

20. Santiago, S.; Zazpe, I.; Cuervo, M.; Martínez, J. A. «Perinatal and parental determinants of childhood overweight in 6-12 years old children». Nutrición Hospitalaria. 2012 April; 27(2).

21. Romero-Velarde, et ál. «Prevalence of metabolic syndrome and associated factors in children and adolescents with obesity». Revista Médica del Instituto Mexicano del Seguro Social. 2016 October; 54(5): 268-75.

22. Descamps O. S; Tarantino, E.; Guilmot, P. F. «Does FTO have a paradoxical effect in fetal life?» BMC Genetics. 2014; 15(1): 384-401.

23. Danielzik, S.; Langnase, K.; Mast, M.; SpethManN, C.; Muller, M. J. «Impact of parental BMI on the manifestation of overweight 5-7 year old children». Eur J Nutr. 2002; 41:132-38.

24. Scaglioni, S.; Agostoni, C.; Notaris, R. D.; Radaelli, G.; Radice, N.; Valenti, M.; GiovanNINI, M. «Early macronutrient intake and overweight at five years of age». Int J Obes Relat Metab Disord. 2000; 24:777-81.

25. Sorensen, T. I.; Price, R. A.; Stunkard, A. J.; SChulsinger, F. «Genetics of obesity in adult adoptees and their biological siblings». BMJ. 1989 January; 298:87-90.

26. Alison, E. Field, N. «Weight Status in Childhood as a Predictor of Becoming Overweight or Hypertensive in Early Adulthood». North Ameri- 
can Association for the Study of Obesity. 2005 January; $13(1)$.

27. Crespo-Salgado, J. J.; Delgado-Martín, J. L.; Blanco-Iglesias, O.; Aldecoa-Landesa, S. «Guía básica de detección del sedentarismo y recomendaciones de actividad física en atención primaria». Atención Primaria. 2015 Marzo; 47(3): p. 175-83.

28. Instituto de Nutrición de Centroamérica y Panamá, INCAP. «Sobrepeso y obesidad afectan al país». elsalvador.com. Agosto de 2014.
29. Gutiérrez, J. P.; Rivera-Dommarco, J.; Shamah-Levy, T.; Villalpando-Hernández, S.; Franco, A.; Cuevas-Nasu, L. Encuesta Nacional de Salud y Nutrición. Resultados Nacionales. México: Instituto Nacional de Salud Pública (MX), Cuernavaca; 2012.

30. Soriano, M.; Kann, L.; Kinchen, S.; Razeghi, G.; Contreras, A. Encuesta Mundial de Salud, resultados El Salvador. OMS. San Salvador: Ministerio de Salud, San Salvador; 2013. 